\title{
BMJ Open Non-invasive ventilation versus oxygen therapy after extubation in patients with obesity in intensive care units: the multicentre randomised EXTUB-OBESE study protocol
}

Audrey De Jong, ${ }^{1}$ Helena Huguet, ${ }^{2}$ Nicolas Molinari, ${ }^{3}$ Samir Jaber (D) ${ }^{1}$

To cite: De Jong A, Huguet $\mathrm{H}$, Molinari $\mathrm{N}$, et al. Non-invasive ventilation versus oxygen therapy after extubation in patients with obesity in intensive care units: the multicentre randomised EXTUB-OBESE study protocol. BMJ Open 2022;12:e052712. doi:10.1136/ bmjopen-2021-052712

- Prepublication history and additional supplemental material for this paper are available online. To view these files, please visit the journal online (http://dx.doi.org/10.1136/ bmjopen-2021-052712).

Received 24 April 2021 Accepted 24 December 2021

Check for updates

(c) Author(s) (or their employer(s)) 2022. Re-use permitted under CC BY-NC. No commercial re-use. See rights and permissions. Published by BMJ.

${ }^{1}$ Département d'Anesthésie Réanimation B PhyMedExp, University Hospital Centre Montpellier, Montpellier, Languedoc-Roussillon, France ${ }^{2}$ Clinical research department of Montpellier university hospital, University Hospital Centre Montpellier, Montpellier Languedoc-Roussillon, France ${ }^{3}$ IMAG, CNRS, Univ Montpellier, University Hospital Centre Montpellier, Montpellier, Languedoc-Roussillon, France

Correspondence to

Dr Samir Jaber;

s-jaber@chu-montpellier.fr

\section{ABSTRACT}

Introduction Patients with obesity are considered to be at high risk of acute respiratory failure (ARF) after extubation in intensive care unit (ICU). Compared with oxygen therapy, non-invasive ventilation (NIV) may prevent ARF in high-risk patients. However, these strategies have never been compared following extubation of critically ill patients with obesity. Our hypothesis is that NIV is associated with less treatment failure compared with oxygen therapy in patients with obesity after extubation in ICU.

Methods and analysis The NIV versus oxygen therapy after extubation in patients with obesity in ICUs protocol (EXTUB-obese) trial is an investigatorinitiated, multicentre, stratified, parallel-group unblinded trial with an electronic system-based randomisation. Patients with obesity defined as a body mass index $\geq 30 \mathrm{~kg} / \mathrm{m}^{2}$ will be randomly assigned in the 'NIV-group' to receive prophylactic NIV applied immediately after extubation combined with high-flow nasal oxygen (HFNO) or standard oxygen between NIV sessions versus in the 'oxygen therapy group' to receive oxygen therapy alone (HFNO or standard oxygen,). The primary outcome is treatment failure within the 72 hours, defined as reintubation for mechanical ventilation, switch to the other study treatment, or premature study-treatment discontinuation (at the request of the patient or for medical reasons such as gastric distention). The single, prespecified, secondary outcome is the incidence of ARF until day 7. Other outcomes analysed will include tracheal intubation rate at day 7 and day 28 , length of ICU and hospital stay, ICU mortality, day 28 and day 90 mortality.

Ethics and dissemination The study project has been approved by the appropriate ethics committee 'Comité-de-Protection-des-Personnes lle de FranceV-19.04.05.70025 Cat2 2019-A00956-51'. Informed consent is required. The results will be submitted for publication in a peer-reviewed journal and presented at one or more scientific conferences. If use of NIV shows positive effects, teams (medical and surgical) will use NIV following extubation of critically ill patients with obesity.

Trial registration number NCT04014920.
Strengths and limitations of this study

- The broad inclusion criteria and the high number of participating intensive care units will increase generalisability of the study.

- The large sample size will provide the opportunity to examine strata and subgroups of interest.

- The double randomisation with stratification will allow to balance groups limiting the confounding factors and to compare both non-invasive ventilation with oxygen therapy, and high-flow nasal oxygen with standard oxygen.

- The main endpoint is a composite criterion that has been previously validated in one large multicentre randomised controlled trial.

- A limitation of this study is that the nature of the study intervention does not allow blinding.

\section{INTRODUCTION}

\section{Background and rationale}

This manuscript was written in accordance with the Standard Protocol Items: Recommendations for Interventional Trials (SPIRIT) guidelines. ${ }^{1}$

Mechanical ventilation is the artificial support most used in intensive care unit (ICU). ${ }^{2}$ If weaning and extubation (removal of the tracheal intubation tube) is successful in approximately $80 \%-90 \%$ of ICU patients, $10 \%-20 \%$ will develop acute respiratory failure (ARF) in the days following extubation. ${ }^{34}$ This incidence is higher in some selected subgroups of patients with underlying lung disease (patients with obesity, chronic obstructive pulmonary disease, elderly, heart failure, postoperative cardiothoracic and/or abdominal surgery ....)..$^{5-7}$

The management of postextubation ARF combines aetiological treatment associated with ventilatory support which usually requires the use of new endotracheal intubation to 
deliver 'invasive' mechanical ventilation, associated with excess morbidity and mortality. ${ }^{89}$

Obesity is associated with excess morbidity and longer length of mechanical ventilation compared with the general population. ${ }^{70}$ Effect of obesity on mortality is controversial, ${ }^{11}$ some studies suggesting a protective or neutral effect of obesity, ${ }^{12}$ named 'obesity paradox'. ${ }^{13}$ At the ventilatory level, several combined pathophysiological changes contribute to an increased incidence of respiratory complications. ${ }^{711}$

For over 20 years, non-invasive ventilation (NIV) has been used to prevent ('preventive NIV') and cure ('curative NIV') ARF in ICU patients. ${ }^{14}{ }^{15}$ An alternative to NIV is the administration of oxygen therapy via standard oxygen or high-flow nasal oxygen (HFNO) ${ }^{16}{ }^{17}$ In an observational study of 124 patients, El Sohl et al $l^{18}$ showed a $16 \%$ absolute risk reduction of ARF using NIV compared with standard oxygen following extubation.

More recently, HFNO has been developed. Highflow rates reduce the dilution of inhaled oxygen and allow precise distribution of $\mathrm{FiO} 2$ throughout the inspiratory phase by adapting the peak flow rate to the patient. ${ }^{17} 19$ High oxygen flow can also have a washing effect on the dead space of the nasopharynx. In addition, a flow-dependent effect helps to generate a continuous positive end-expiratory pressure (PEEP) ${ }^{20}$ Finally, the use of a high level of humidity could prevent alterations of the ciliated epithelium of the respiratory tract, maintain the activity of the muco-ciliary system, and facilitate the elimination of secretions. ${ }^{21}$ In a post hoc analysis of a large trial of 830 postoperative thoracic patients, ${ }^{22}$ it was shown that among the 272 patients with obesity (mean body mass index (BMI) of $34 \mathrm{~kg} / \mathrm{m}^{2}$ ), NIV was not superior to HFNO, with treatment failure occurring in $15 \%$ and $13 \%$ in NIV and HFNO groups respectively. Moreover, in 155 post cardiac surgery patients with obesity, Corley et $a l^{23}$ compared HFNO with standard oxygen to prevent ARF, without showing any difference.

However, none of these studies compared simultaneously the most recent devices available: NIV, HFNO and standard oxygen, nor their association. ${ }^{24}$ HFNO is now often used, ${ }^{25}$ and the PEEP issued by HFNO is much lower than that issued by NIV. The benefit of NIV compared with oxygen therapy (HFNO or standard oxygen) after extubation of critically ill patients with obesity has never been studied.

In this multicentre, randomised, controlled, interventional study in mechanically ventilated critically ill patients with obesity, we will test the hypothesis that NIV (associated with HFNO or standard oxygen between NIV trials) could reduce the rate of treatment failure in comparison with oxygen therapy alone continuously administered (HFNO or standard oxygen) in patients with obesity within 72 hours after extubation in an ICU.

\section{Objectives}

Primary objective. To determine whether NIV could reduce the rate of treatment failure in comparison with oxygen therapy within the 72 hours after extubation of critically patients with obesity, defined as reintubation for mechanical ventilation, switch to the other study treatment, or premature study-treatment discontinuation (at the request of the patient or for medical reasons such as gastric distention).

Secondary objectives. To determine whether NIV could reduce the rate of ARF at day 7 and other secondary outcomes in comparison with oxygen therapy.

Stratified and subgroups analyses according to variable of stratification (length of mechanical ventilation $<48$ hours vs $\geq 48$ hours, type of admission (medical vs surgical), centre and patients characteristics will be done.

The main hypothesis is that NIV (associated with HFNO or standard oxygen) could reduce the rate of treatment failure in comparison with oxygen therapy alone (HFNO or standard oxygen) in patients with obesity within the 72 hours after extubation in ICU.

\section{Trial design}

The NIV versus oxygen after extubation in patients with obesity in ICUs (EXTUB-obese) trial is an investigatorinitiated, multicentre, stratified, parallel-group unblinded trial with an electronic system-based randomisation.

Patients will be randomly assigned (first randomisation) to receive NIV (experimental group) or to receive oxygen therapy (control group). A second randomisation of both groups will determine the type of oxygen received in each group: (1) NIV with HFNO between sessions, or NIV with standard oxygen between sessions (experimental group) or (2) HFNO or standard oxygen (control group, figure 1).

\section{Consort diagram}

Figure 1 shows the CONSORT diagram of the EXTUBobese trial.

\section{METHODS: PARTICIPANTS, INTERVENTIONS AND OUTCOMES Study setting}

This study will take place in 35 ICUs in France, belonging to a research network that specialises in the management of critically ill patients and has a particularly high level of expertise in respiratory care strategies. ${ }^{26}$

\section{Eligibility criteria}

Inclusion criteria

Patients must be present in the ICU, adult (age $\geq 18$ years), covered by public health insurance, with written informed consent from the patient or proxy (if present) before inclusion or once possible if the patient has been included in an emergency context, with obesity defined by a BMI $\geq 30 \mathrm{~kg} / \mathrm{m}^{2}$ the day of extubation and require extubation in ICU after a length of mechanical ventilation of more than 6 hours.

\section{Exclusion criteria}

Patients fulfilling one or more of the following criteria will not be included: hypercapnia with a formal indication for 


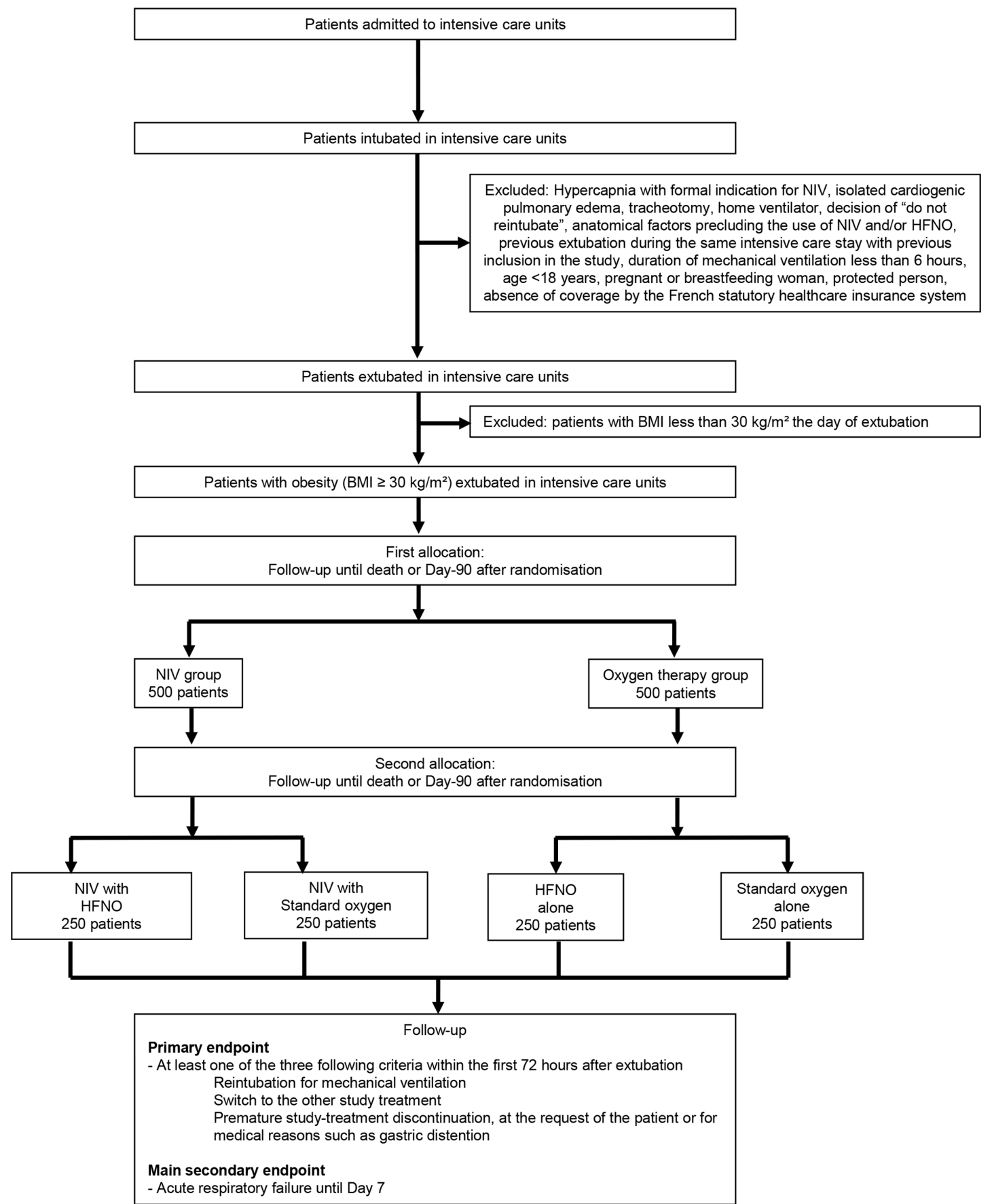

Figure 1 Consort diagram of the EXTUB obese trial. BMI, body mass index; HFNO, high-flow nasal oxygen; NIV, non-invasive ventilation.

NIV (arterial carbon dioxide pressure $(\mathrm{PaCO} 2 \geq 50 \mathrm{~mm}$ $\mathrm{Hg}$ )), isolated cardiogenic pulmonary oedema (formal indication for NIV, patients with pulmonary oedema associated with another ARF aetiology can be included), tracheotomy, home ventilator, end-of-life decision with decision of 'do not reintubate', anatomical factors precluding the use of NIV and/or HFNO, previous extubation during the same ICU stay with previous inclusion in the study, duration of mechanical ventilation less than 6 hours, age $<18$ years, pregnant or breastfeeding woman, protected person, refusal of study participation or to pursue the study by the patient, absence of coverage by the French statutory healthcare insurance system.

\section{Outcomes}

Primary outcome

Primary outcome variable is treatment failure within the 72 hours after extubation, defined as reintubation for mechanical ventilation, switch to the other study treatment, or premature study-treatment discontinuation (at the request of the patient or for medical reasons such as gastric distention). ${ }^{5}$ 
For the primary analysis, comparing NIV to oxygen therapy, switch to the other study treatment will be defined as switch from oxygen therapy to NIV. Premature study-treatment discontinuation will be defined as discontinuation of NIV or HFNO at the request of the patient before completion of one session of NIV of at least $30 \mathrm{~min}$ in the NIV group or before 12 hours of HFNO in the oxygen therapy group or for medical reasons such as gastric distention.

For the secondary analysis, comparing also HFNO to standard oxygen, switch to the other study treatment will also be defined as switch from oxygen therapy to HFNO.

\section{Main secondary outcome}

The single, prespecified, secondary outcome is incidence of ARF until day 7.

ARF during the first 7 days will be defined by two criteria among the following: ${ }^{27}$

1. Hypercapnia $\left(\mathrm{PaCO}_{2}>45 \mathrm{~mm} \mathrm{Hg}\right)$ with respiratory acidosis (arterial $\mathrm{pH} \leq 7.35$ ).

2. Modification of mental state and / or of conscience level (agitation or encephalopathy).

3. Decrease of $\mathrm{SpO}_{2}<90 \%$ or $\mathrm{PaO}_{2}<55 \mathrm{~mm} \mathrm{Hg}$ despite a $\mathrm{FiO}_{2}>50 \%$ and a flow $>10 \mathrm{~L} / \mathrm{min}$.

4. Haemodynamic instability, with a systolic arterial pressure $<70 \mathrm{~mm} \mathrm{Hg}$ during more than $30 \mathrm{~min}$ despite sufficient fluid loading and/or the use of vasopressors.

5. Abundant secretions and ineffective cough.

6 . Respiratory rate $>35 / \mathrm{min}$, with signs of respiratory distress.

These criteria will be collected each day until day 7 .

\section{Exploratory outcomes}

Other outcomes will be evaluated as exploratory clinical outcomes:

- Oxygenation evaluated by the Pressure of Arterial Oxygen to Fractional Inspired Oxygen Concentration $(\mathrm{PaO} 2 / \mathrm{FiO} 2)$ ratio until day 7.

- Organ failure until day 7 assessed with the sequential organ failure assessment (SOFA) score.

- Tracheal intubation rate at day 7, day 14 or day 28 .

- Length of stay in ICU and in hospital.

- ICU, day 28 and day 90 mortality rates.

\section{Safety/adverse outcomes}

- Death during the NIV, HFNO or standard oxygen sessions.

- Cardiac arrest during the NIV, HFNO or standard oxygen sessions.

Subanalysis of reintubation occurrences according to prespecified criteria ${ }^{28}$ will be performed.

\section{Interventions}

All consecutive extubation procedures will be screened for inclusion. A spontaneous breathing trial before extubation will not be mandatory and will be left at the clinician discretion. The decision of extubation will be left to the discretion of the physician. When an extubation is planned by the physician in charge of a patient with obesity, the patient will receive two consecutive randomisations. A first randomisation will be performed to allocate the patient in the experimental group to receive intermittent NIV trials or in the oxygen therapy group to receive continuous oxygen therapy. A second consecutive randomisation will determine the type of oxygen received in each group. For the experimental group, the second randomisation will determine the type of oxygen received between NIV trials (HFNO or standard oxygen). For the oxygen therapy group, the second randomisation will determine the type of oxygen continuously administered (HFNO or standard oxygen). NIV will not be used in the oxygen therapy group, except in the case of rescue therapy in the case of ARF and at the physician's request. HFNO will not be used in patients of both NIV and oxygen therapy group who will receive standard oxygen after the second randomisation (figure 1), except in the case of rescue therapy in the case of ARF and at the physician's request.

In the experimental group, the first NIV session will be offered to the patient within $30 \mathrm{~min}$ following extubation. The NIV system will first be explained to the patient by the physician or nurse and positioned at bedside. The mask will be chosen according to patient's facial morphology. The mask will be placed and adjusted to avoid leaks. Recommended PEEP value will be set to $10 \mathrm{cmH}_{2} \mathrm{O}$ (and adapted between 5 and $10 \mathrm{cmH}_{2} \mathrm{O}$ depending on tolerance) and value of pressure support (PS) will be set to obtain a respiratory rate between 20 and 30 breaths per minute (bpm) and an expired tidal volume in-between 6 and $8 \mathrm{~mL} / \mathrm{kg}$ of ideal body weight. The recommended length of the intermittent NIV sessions will be standardised as follows: sessions of 30-60 min spread through the day and night for a cumulated time of at least 4 hours with no upper limit during the first 24 hours. NIV weaning will start 24 hours after extubation, if respiratory rate is stable and less than 25/ min with a $\mathrm{PaO}_{2} / \mathrm{FiO}_{2}$ ratio of more than $200 \mathrm{~mm} \mathrm{Hg}$ and a $\mathrm{PaCO}_{2}$ less than $45 \mathrm{~mm} \mathrm{Hg}$ on the blood gases at H24. Between NIV sessions, patients will receive oxygen therapy with the same methods as the oxygen therapy group, with HFNO or standard oxygen.

In both groups, the second randomised device (oxygen therapy device) will be HFNO or standard oxygen.

HFNO will be administered at a flow of $50 \mathrm{~L} / \mathrm{min}$ during the first 24 hours (can be reduced with patient improvement/tolerance to $30 \mathrm{~L} / \mathrm{min}$ if required), with an $\mathrm{FiO} 2$ set to target $\mathrm{SpO} 2 \geq 94 \%$, which may in some patients reduce to an $\mathrm{FiO} 2$ of $21 \%$. Patients administered standard oxygen will receive this therapy only in the case where $\mathrm{SpO} 2 \leq 94 \%$. We chose the $\mathrm{SpO} 2$ threshold of $94 \%$ as it is "standard practices" in the majority of the hospitals participating to the study.

After 24 hours, the device will be pursued if the patient still needs oxygen, until the discharge from ICU or the absence of need of oxygen. The patients will be followed during their ICU stay and hospital stay until discharge or death. The follow-up will be stopped at 3 months. 
Table 1 Participant timeline

\begin{tabular}{llll}
\hline Item & Screening/baseline & & \\
\hline & Visit 1 & Visit 2 & Visit 3 \\
\hline Date & H0 & H72 & Day 28 or ICU discharge \\
\hline Clinical evaluation & $\mathbf{X}$ & $\mathbf{X}$ & $\mathbf{X}$ \\
Informed consent & $\mathbf{X}$ & & \\
Medical history & $\mathbf{X}$ & & \\
Demography & $\mathbf{X}$ & & \\
Physical examination & $\mathbf{X}$ & $\mathbf{X}$ & $\mathbf{X}$ \\
Vital signs & $\mathbf{X}$ & $\mathbf{X}$ & $\mathbf{X}$ \\
Routine laboratory testing $\dagger$ & $\mathbf{X}$ & $\mathbf{X}$ & $\mathbf{X}$ \\
Experimental treatment $^{*}$ & $\mathbf{X}$ & $\mathbf{X}$ & $\mathbf{X}$ \\
Endpoints evaluation $^{\circ}$ & $\mathbf{X}$ & $\mathbf{X}$ & $\mathbf{X}$ \\
Adverse events recording $^{*}$ & $\mathbf{X}$ & $\mathbf{X}$ & $\mathbf{X}$ \\
\hline
\end{tabular}

*Includes haemodynamic parameters (arterial pressure, heart rate, vasopressors use), respiratory rate, ventilatory parameters (respiratory rate and pulse oxymetry).

†Arterial blood gases, as usually performed for the daily patient care during the first 72 hours if an arterial catheter was in place. Supplementary blood gases will be done according to the clinical state of the patient. Blood gases will be also done before the reintubation if an acute respiratory failure following extubation occurs.

$\mathrm{ICU}$, intensive care unit.

Final decision of reintubation will be taken by the physician according to prespecified criteria $^{28}$ : respiratory or cardiac arrest, respiratory pauses with loss of consciousness or gasping for air, massive aspiration, persistent inability to clear respiratory secretions, heart rate of less than 50/min with loss of alertness, and severe haemodynamic instability without response to fluid and vasoactive drugs.

\section{Participant timeline}

Participant timeline is presented in table 1 and figure 2.

\section{Sample size}

Two intermediate analyses will be performed after inclusion of 250 and 500 patients (stop for efficacy or safety). Assuming the overall $p$ value for the trial is 0.05 , the $p$ value threshold is 0.001 for the two interim analyses and 0.05 for the final analysis (Haybittle-Peto boundary).

Based on a $12 \%$ composite endpoint rate in the oxygen group (Free rea study ${ }^{4}$ and a decrease of $50 \%$ of the composite endpoint rate to $6 \%$ in the NIV group, ${ }^{18}$ with an alpha risk set at $5 \%$, to obtain a $80 \%$ power for demonstrating superiority for the primary outcome, we need 954 patients (477 in each group) to demonstrate a superiority of NIV to oxygen therapy. In order to take into account lost to follow-up and intubation for surgical procedures without criteria of ARF, we will include 1000 patients.

\section{Recruitment}

Patients are expected to be included during a 2-year inclusion period starting October 2019. Among the 35

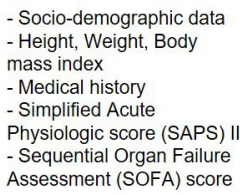

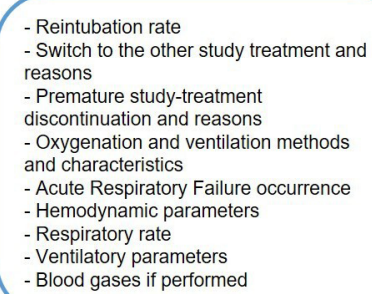

- Length of oxygenation and noninvasive ventilation

- Length of stay in

intensive care unit

- Mortality rate at Day

28 and in intensive care

unit

Reintubation rate
Length of stay in hospita

Mortality rate at Day 90 and in hospital

Figure 2 Timeline of data collection. 
participating centres, each centre would need to include 1-2 patients per month during the 24 months-study period.

March 2019-September 2019: Protocol, approvals from ethics committee, and trial tool development (case report form, randomisation system).

October 2019-September 2021: Inclusion of patients.

October 2021-December 2021: Cleaning and closure of the database.

January 2022-September 2022: Data analyses, writing of the manuscript and submission for publication.

\section{METHODS: ASSIGNMENT OF INTERVENTIONS Allocation and sequence generation}

Randomisation will be managed by the clinical research unit of Montpellier University Hospital with Capture System software (Ennov Clincalt, randomisation module). The randomisation will be centralised and available online. It will be stratified on centre, ${ }^{29}{ }^{30}$ length of mechanical ventilation ( $<48$ hours vs $\geq 48$ hours) and on type of admission (medical vs surgical), balanced with a 1:1 ratio and minimisation. The randomisation will be performed from 1 hour before extubation to $30 \mathrm{~min}$ after extubation. It was decided to authorise the physician to perform randomisation in the $30 \mathrm{~min}$ following extubation in order to be still able to include patients if the physician had forgotten before extubation.

\section{Blinding}

Given the nature of the devices, a blinded design is not possible for the investigator and associate investigator. The methodologist will be blinded to the group.

\section{METHODS: DATA COLLECTION, MANAGEMENT AND ANALYSIS Data collection and management}

Data will be collected and recorded on electronic case report forms by trained local research coordinators or physicians. Patients will receive standard ICU monitoring consisting of ECG analysis, peripheral oxygen saturation, and a noninvasive blood pressure cuff.

The day of extubation (from $\mathrm{H} 0$ to $\mathrm{H} 24$ beginning from the time of extubation), the following data will be collected: demographic data, Simplified Acute Physiology Score (SAPS) II, length of stay in ICU before inclusion, length of mechanical ventilation before inclusion, reason for intubation, comorbidities, haemodynamic parameters (arterial pressure, heart rate, vasopressors use), ventilatory parameters (respiratory rate and $\mathrm{SpO}_{2}$ ), spontaneous breathing trial characteristics (t-tube or PS, if performed), oxygenation and if performed NIV characteristics and the SOFA score.

From day 1 to day 7 , the investigator or designated study personnel will record the criteria for the main outcome (reintubation and main reason for reintubation, oxygenation method (continued, stopped, changed)) and for the secondary outcomes (ARF, oxygenation, mortality).
They will also assess reason for intubation, haemodynamic variables (arterial pressure, heart rate, vasopressors use), ventilatory variables (respiratory rate and $\mathrm{SpO}_{2}$ ), oxygenation and if performed NIV characteristics.

Length of stay in ICU will be evaluated. At ICU discharge, day 28 and day 90 , mortality rate will be evaluated.

\section{Statistical methods}

The statistical analysis will incorporate all the elements required by the CONSORT statement for nonpharmacological interventions. Statistical analysis will be performed in an intention-to-treat population, including all the randomised patients except patients who withdraw their consent, were protected or not covered by the French statutory healthcare insurance system, worsened just before extubation and were not extubated, or had no main outcome recorded on electronic case report form.

Then, a per-protocol analysis will be performed, excluding the patients with reintubation for surgical procedures without criteria of ARF, with BMI less than $30 \mathrm{~kg} / \mathrm{m}^{2}$ or with home ventilator.

All analyses will be conducted by the medical statistical department of the Montpellier University Hospital using statistical software (SAS, V.9.4; SAS Institute and R, V.3.6.2). A two-sided $p<0.05$ will be considered to indicate statistical significance.

\section{Description of the patient groups at baseline}

The baseline features of the overall population and of each group will be described. Categorical variables will be reported as frequencies and percentages and continuous variables as either means with SDs or medians with IQRs.

\section{Primary analysis}

Uncorrected $\chi^{2}$ test will be used for primary outcome analysis (comparison of the composite criteria at H72 combining reintubation for invasive mechanical ventilation, the switch to the other study treatment or the premature study treatment discontinuation).

A logistic regression will be used for the analysis of the primary outcome with OR of failure calculation, before and after adjustment on confounding variables despite the randomisation. A supplementary analysis on the primary outcome will be done for the time without treatment failure, per study group, using the log rank test. Unadjusted Kaplan-Meier curves with respect to the primary outcome for the two groups will be performed to see if both curves do not cross each other, that is, the assumption of proportionality of the Cox model is not breached. In this case, a Cox model will be performed for the time without treatment failure, before and after adjustment. Covariates will be defined as binary variables and continuous variables dichotomised according to their median tested in the model, and will be selected a priori and limited according to the number of events of primary outcome (reason for intubation, previous respiratory disease and SOFA score) and then presented as adjusted ORs or adjusted HRs with $95 \%$ CIs. A centre effect will 
be checked using a mixed effect model, considering the centre both as a random and then a fixed variable. Interactions between variables will be tested.

Then, unadjusted stratified and subgroups analyses according to variable of stratification (length of mechanical ventilation $<48$ hours vs $\geq 48$ hours, type of admission (medical vs surgical), centre) and patients characteristics will be done on the primary outcome and reintubation rate.

A centre effect will be checked using a mixed effect model, considering the centre both as a random and then a fixed variable. Interactions between variables and time will be tested.

\section{Secondary analyses}

Continuous outcomes will be compared with the Student's t-test or Mann-Whitney rank-sum test according to the conditions of application and categorical variables with the $\chi^{2}$ test or the Fisher's exact test, according to the conditions of application. Then, stratified and subgroups analyses according to variable of stratification (length of mechanical ventilation $<48$ hours vs $\geq 48$ hours, type of admission (medical vs surgical), centre), type of oxygenation (second randomisation) and patients characteristics will be done.

\section{Interim analysis}

This trial will be planned with two interim analyses after the observation of the primary outcome of 250 and 500 patients. The interim analysis will be planned for early stopping of the study owning to safety (as defined by mortality within 7 days) or efficiency on the primary outcome after the first 250 and 500 patients included assuming the overall $p$ value for the trial is 0.05 , $p$ value threshold is 0.001 for the two interim analyses and 0.05 for the final analysis (Haybittle-Peto boundary).

\section{Handling of missing data}

Based on prior trials in similar settings, we anticipate less than $5 \%$ missing data for the primary outcome. For the primary analysis, missing data will not be imputed.

\section{Corrections for multiple testing}

We have prespecified a single primary analysis of a single primary outcome. For the exploratory outcomes, a false discovery rate method ${ }^{31}$ will be used.

\section{METHODS: MONITORING \\ Data monitoring}

Before the start of patient recruitment, all physicians and other healthcare workers in the ICUs will attend formal training sessions on the study protocol and data collection.

The physicians and a clinical research nurse and/or clinical research assistant are in charge of daily patient screening and inclusion, ensuring compliance with the study protocol and collecting the study data, with blinded assessment.
Harms

Since the devices used (NIV, HFNO, standard oxygen) are already marketed and used in current clinical practice, the use of these devices does not seem likely to generate a significant risk during this protocol.

Regarding the vigilance of the project, the responsibilities of the investigator and sponsor, the reporting of serious adverse events (AE), annual safety reports will be monitored and carried out in accordance with regulations.

Complete and appropriate data on all AEs experienced during the clinical trial will be recorded on the $\mathrm{AE}$ form of the case report form on an ongoing basis for the duration of the study. Each AE report shall include a description of the event, an assessment of its seriousness according to the criteria listed above, its duration, intensity, relationship to the study treatment, other causality factors (if any), any concomitant medication dispensed, actions taken with the study device or other therapeutic interventions and outcome at the end of the observation period.

For each $\mathrm{AE}$, a separate $\mathrm{AE}$ form will be filled in.

\section{Ethics and dissemination}

Research ethics approval

This research involving humans will be conducted in compliance with French 'Loi no 2012-300 du 5 mars 2012 relative aux recherches impliquant la personne humaine (Loi Jardé), 'Loi No 78-17 du 6 janvier 1978 modifiée relative à l'Informatique, aux fichiers et aux Libertés').

This study will be conducted in accordance with Good Clinical Practice, as defined by the International Conference on Harmonisation.

The study project has been approved by the ethics committee 'Comité de Protection des Personnes Ile de France V 19.04.05.70025 Cat 2 2019-A00956-51'. The EXTUB obese study is conducted in accordance with the Declaration of Helsinki.

\section{Consent or assent}

Three methods of consent will be used, as required by the institutional review board in accordance with the 2013 Declaration of Helsinki. If possible, the patient will be included after written informed consent. However, the patient often cannot understand information given because of underlying disease. These patients will be included after written informed consent is provided by next of kin or an emergency procedure (investigator signature) if next of kin is not present. When possible, after recovery, patients will be retrospectively asked for written consent to continue the trial. Informed consent material is available in online supplemental file 1 .

\section{Patient and public involvement}

The development of the research question and outcome measures was not informed by patients' priorities, experience and preferences. Patients were not involved in the design, recruitment and conduct of the study. The 
burden of the intervention will not be assessed by patients themselves. The results will be available for study participants on demand. No systematic disseminating of the results for study participants is planned.

\section{Confidentiality}

Data will be handled according to French law. All original records will be archived at trial sites for 15 years. The clean database file will be anonymised and kept for 15 years.

\section{Declaration of interest}

The study is an investigator-initiated trial. Study promotion is performed by Montpellier University Hospital, Montpellier, France. There is no industry support or involvement in the trial.

\section{Dissemination policy}

Findings will be published in peer-reviewed journals and presented at local, national and international meetings and conferences to publicise and explain the research to clinicians, commissioners and service users. All investigators will have access to the final data set. Participant-level data sets will be made accessible on a controlled access basis.

\section{DISCUSSION}

To the best of our knowledge, the EXTUB OBESE trial is the first pragmatic randomised controlled trial powered to investigate if NIV reduces treatment failure at H72 after extubation of ICU patients with obesity, compared with oxygen therapy (HFNO or standard oxygen).

NIV has proven effective in small observational studies in preventing post-extubation ARF in patients with obesity, in an ICU or postoperative setting. ${ }^{11} 18$ 32-34 Standard oxygen (control group) was the standard practice in past years, however, currently HFNO has become the more common practice, and has proven to be non-inferior to NIV in ARF patients following cardiothoracic surgery and in high-risk patients after extubation in the ICU. ${ }^{522}$

However, a recent published study ${ }^{6}$ was performed to assess NIV in a large population of patients older than 65 years or with underlying chronic cardiac or respiratory disease. In this multicentre, randomised, open-label trial, the authors found that HFNO with NIV, compared with HFNO alone, decreased the rate of reintubation within the first 7 days after extubation in the ICU. It is worth noting that patients with obesity were only included if they had underlying chronic cardiac or respiratory disease, such as obesity hypoventilation syndrome. The current study aims to assess all patients with obesity after a length of invasive mechanical ventilation of at least 6 hours. In this setting of previous studies comparing NIV and standard oxygen showing superiority of NIV, ${ }^{11} 18$ 31-33 and according to the recent study of Thille $e t a l{ }^{6}{ }^{6}$ we chose to design the trial as a superiority trial of NIV over oxygen therapy (including HFNO and standard oxygen). The stratification of randomisation according to the length of mechanical ventilation (less or more than 48 hours) and the type of admission (medical vs surgical), will allow to conclude on several strata of patients with obesity and different severities and profiles.

One of the strengths of the study is that the two consecutive randomisations will allow to balance the groups limiting the confounding factors. Moreover, the double randomisation will allow to compare both NIV with oxygen therapy, and HFNO with standard oxygen, and stratification will allow strata analyses.

One other strength is that the team has extensive experience in performing studies about NIV and HFNO or standard oxygen, including randomised controlled trials. 3528 The research networks involved in several study groups will be used..$^{28} 4302636$ No industry will be involved, and HFNO and NIV are available and widely used in all participating centres, another strength of the study.

One of the limitations is that given the nature of the devices, a blinded design is not possible for the investigator and associate investigator. However, to limit the risk of bias, the methodologist will be blinded to the group.

In conclusion, the EXTUB obese trial is the first investigator initiated pragmatic randomised controlled trial powered to test the hypothesis that NIV is associated with less treatment failure compared with oxygen therapy in patients with obesity within the 72 hours after extubation in an ICU.

\section{Trial status}

The trial has started and is actively enrolling since October 2019.

Contributors ADJ drafted the manuscript together with SJ. SJ designed the study together with ADJ. NM, HH and ADJ wrote the statistical analysis plan and estimated the sample size. All authors (ADJ, HH, NM and SJ) revised the manuscript for important intellectual content and read and approved the final version of the manuscript.

Funding The study is an investigator-initiated trial. Study promoter is National Ministry of Health, France (PHRCN-18-0078 Finess 340780477).

Disclaimer There is no industry support or involvement in the trial.

Competing interests SJ reports receiving consulting fees from Drager, Medtronic, Baxter, Fresenius-Xenios, and Fisher \& Paykel. ADJ reports receiving consulting fees from Medtronic. No potential conflict of interest relevant to this article was reported for other authors.

Patient consent for publication Not applicable.

Provenance and peer review Not commissioned; externally peer reviewed.

Supplemental material This content has been supplied by the author(s). It has not been vetted by BMJ Publishing Group Limited (BMJ) and may not have been peer-reviewed. Any opinions or recommendations discussed are solely those of the author(s) and are not endorsed by BMJ. BMJ disclaims all liability and responsibility arising from any reliance placed on the content. Where the content includes any translated material, BMJ does not warrant the accuracy and reliability of the translations (including but not limited to local regulations, clinical guidelines, terminology, drug names and drug dosages), and is not responsible for any error and/or omissions arising from translation and adaptation or otherwise.

Open access This is an open access article distributed in accordance with the Creative Commons Attribution Non Commercial (CC BY-NC 4.0) license, which permits others to distribute, remix, adapt, build upon this work non-commercially, and license their derivative works on different terms, provided the original work is properly cited, appropriate credit is given, any changes made indicated, and the use is non-commercial. See: http://creativecommons.org/licenses/by-nc/4.0/. 
ORCID iD

Samir Jaber http://orcid.org/0000-0002-7257-8069

\section{REFERENCES}

1 Chan A-W, Tetzlaff JM, Gøtzsche PC, et al. Spirit 2013 explanation and elaboration: guidance for protocols of clinical trials. BMJ 2013;346:e7586.

2 Jaber S, Citerio G, Slutsky AS. Acute respiratory failure and mechanical ventilation in the context of the COVID-19 pandemic: why a special issue in ICM? Intensive Care Med 2020;46:2131-2.

3 Boles J-M, Bion J, Connors A, et al. Weaning from mechanical ventilation. Eur Respir J 2007;29:1033-56.

4 Jaber S, Quintard H, Cinotti R, et al. Risk factors and outcomes for airway failure versus non-airway failure in the intensive care unit: a multicenter observational study of 1514 extubation procedures. Crit Care 2018;22:236.

5 Stéphan F, Barrucand B, Petit P, et al. High-Flow nasal oxygen vs noninvasive positive airway pressure in hypoxemic patients after cardiothoracic surgery: a randomized clinical trial. JAMA 2015;313:2331-9.

6 Thille AW, Muller G, Gacouin A, et al. Effect of Postextubation high-flow nasal oxygen with noninvasive ventilation vs highflow nasal oxygen alone on Reintubation among patients at high risk of extubation failure: a randomized clinical trial. JAMA 2019;322:1465-75.

7 De Jong A, Wrigge H, Hedenstierna G, et al. How to ventilate obese patients in the ICU. Intensive Care Med 2020;46:2423-35.

8 Mithoefer JC, Karetzky MS, Mead GD. Oxygen therapy in respiratory failure. N Engl J Med 1967;277:947-9.

9 Chanques G, Constantin J-M, Devlin JW, et al. Analgesia and sedation in patients with ARDS. Intensive Care Med 2020;46:2342-56.

10 Akinnusi ME, Pineda LA, El Solh AA. Effect of obesity on intensive care morbidity and mortality: a meta-analysis. Crit Care Med 2008;36:151-8.

11 De Jong A, Chanques G, Jaber S. Mechanical ventilation in obese ICU patients: from intubation to extubation. Crit Care 2017;21:63.

12 De Jong A, Verzilli D, Sebbane M, et al. Medical versus surgical ICU obese patient outcome: a Propensity-Matched analysis to resolve clinical trial controversies. Crit Care Med 2018;46:e294-301.

13 De Jong A, Jung B, Chanques G, et al. Obesity and mortality in critically ill patients: another case of the Simpson paradox? Chest 2012;141:1637-8.

14 Jaber S, Chanques G, Jung B. Postoperative noninvasive ventilation. Anesthesiology 2010;112:453-61.

15 Demoule A, Chevret S, Carlucci A, et al. Changing use of noninvasive ventilation in critically ill patients: trends over 15 years in francophone countries. Intensive Care Med 2016;42:82-92.

16 Chanques G, Riboulet F, Molinari N, et al. Comparison of three high flow oxygen therapy delivery devices: a clinical physiological crossover study. Minerva Anestesiol 2013;79:1344-55.

17 Rochwerg B, Einav S, Chaudhuri D, et al. The role for high flow nasal cannula as a respiratory support strategy in adults: a clinical practice guideline. Intensive Care Med 2020;46:2226-37.

18 El-Solh AA, Aquilina A, Pineda L, et al. Noninvasive ventilation for prevention of post-extubation respiratory failure in obese patients. Eur Respir J 2006;28:588-95.

19 Ricard J-D, Roca O, Lemiale V, et al. Use of nasal high flow oxygen during acute respiratory failure. Intensive Care Med 2020;46:2238-47.
20 Parke R, McGuinness S, Eccleston M. Nasal high-flow therapy delivers low level positive airway pressure. $\mathrm{Br} J$ Anaesth 2009;103:886-90.

21 Chanques G, Constantin J-M, Sauter M, et al. Discomfort associated with underhumidified high-flow oxygen therapy in critically ill patients. Intensive Care Med 2009;35:996-1003.

22 Stéphan F, Bérard L, Rézaiguia-Delclaux S, et al. High-Flow nasal cannula therapy versus intermittent noninvasive ventilation in obese subjects after cardiothoracic surgery. Respir Care 2017;62:1193-202.

23 Corley A, Bull T, Spooner AJ, et al. Direct extubation onto high-flow nasal cannulae post-cardiac surgery versus standard treatment in patients with a $\mathrm{BMI} \geq 30$ : a randomised controlled trial. Intensive Care Med 2015;41:887-94.

24 Jung B, Vaschetto R, Jaber S. Ten tips to optimize weaning and extubation success in the critically ill. Intensive Care Med 2020;46:2461-3.

25 Papazian L, Corley A, Hess D, et al. Use of high-flow nasal cannula oxygenation in ICU adults: a narrative review. Intensive Care Med 2016;42:1336-49.

26 Jaber S, Rollé A, Godet T, et al. Effect of the use of an endotracheal tube and Stylet versus an endotracheal tube alone on first-attempt intubation success: a multicentre, randomised clinical trial in 999 patients. Intensive Care Med 2021;47:In Press.

27 Frat J-P, Thille AW, Mercat A, et al. High-Flow oxygen through nasal cannula in acute hypoxemic respiratory failure. $N$ Engl J Med 2015;372:2185-96.

28 Jaber S, Lescot T, Futier E, et al. Effect of noninvasive ventilation on tracheal Reintubation among patients with hypoxemic respiratory failure following abdominal surgery: a randomized clinical trial. JAMA 2016;315:1345-53.

29 Quintard H, l'Her E, Pottecher J, et al. Intubation and extubation of the ICU patient. Anaesth Crit Care Pain Med 2017;36:327-41.

30 De Jong A, Molinari N, Terzi N, et al. Early identification of patients at risk for difficult intubation in the intensive care unit: development and validation of the MACOCHA score in a multicenter cohort study. Am J Respir Crit Care Med 2013;187:832-9.

31 Benjamini Y, Hochberg Y. Controlling the false discovery rate: a practical and powerful approach to multiple testing. Journal of the Royal Statistical Society: Series B 1995;57:289-300 https://www. jstor.org/stable/2346101

32 Pépin JL, Timsit JF, Tamisier R, et al. Prevention and care of respiratory failure in obese patients. Lancet Respir Med 2016;4:407-18.

33 Neligan PJ, Malhotra G, Fraser M, et al. Continuous positive airway pressure via the Boussignac system immediately after extubation improves lung function in morbidly obese patients with obstructive sleep apnea undergoing laparoscopic bariatric surgery. Anesthesiology 2009;110:878-84.

34 Zoremba M, Kalmus G, Begemann D, et al. Short term non-invasive ventilation post-surgery improves arterial blood-gases in obese subjects compared to supplemental oxygen delivery - a randomized controlled trial. BMC Anesthesiol 2011;11:10.

35 Futier E, Paugam-Burtz C, Godet T, et al. Effect of early postextubation high-flow nasal cannula vs conventional oxygen therapy on hypoxaemia in patients after major abdominal surgery: a French multicentre randomised controlled trial (opera). Intensive Care Med 2016;42:1888-98.

36 Jaber S, Rolle A, Jung B, et al. Effect of endotracheal tube plus stylet versus endotracheal tube alone on successful firstattempt tracheal intubation among critically ill patients: the multicentre randomised STYLETO study protocol. BMJ Open 2020;10:e036718. 